\title{
Prevalence and risk factors for age-related cataract in Sweden
}

\author{
Magnus Hugosson ${ }^{\mathrm{a}}$ and Curt Ekström ${ }^{\mathrm{b}}$ (ID

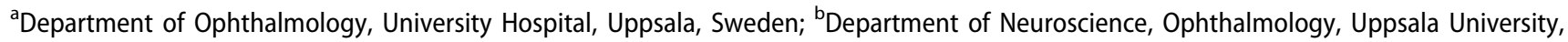 \\ Uppsala, Sweden
}

\begin{abstract}
Background: Cataract is a major cause of visual impairment worldwide. There is a paucity of prevalence studies from Sweden. Therefore, we report the prevalence of cataract and its risk factors in a population-based study of older adults in Sweden.

Methods: The Tierp Glaucoma Survey was conducted in the municipality of Tierp, Sweden, including 760 subjects aged 65-74 years. The presence of cataract was determined based on retroillumination, with lens opacities evident on slit-lamp examination. To assess risk factors for cataract, odds ratios (ORs) were calculated, adjusted for age and gender.

Results: A total of 234 individuals were found to have cataract, 12 of whom had undergone cataract surgery. The prevalence adjusted for nonparticipation was 31.5\% (95\% confidence interval [Cl] 29.4-33.6), 35.2\% (95\% Cl 28.7-41.8) in females and $26.2 \%(95 \% \mathrm{Cl} 19.8-32.6)$ in males. Cataract was associated with age $\geq 70$ years (OR 1.93; $95 \% \mathrm{Cl} 1.41-2.64$ ), female gender (OR $1.54 ; 95 \% \mathrm{Cl} 1.12-2.11$ ), and myopia (OR 2.3; $95 \% \mathrm{Cl} 1.16-3.56$ ), while pseudoexfoliation, smoking, diabetes, hypertension, and ischaemic heart disease were not.

Conclusion: Nearly one-third of the sample were estimated to have lens opacities, or had undergone cataract surgery, making cataract a frequent disorder of older age. The study provided further evidence that increasing age, female gender, and myopia are associated with cataract.
\end{abstract}

ARTICLE HISTORY

Received 28 April 2020

Revised 18 June 2020

Accepted 23 July 2020

\section{KEYWORDS}

Cataract; epidemiology; population survey; prevalence; risk factor

\section{Introduction}

Cataract remains a major cause of visual impairment worldwide (1). However, in many countries cataract surgery is easily available. In fact, cataract surgery is one of the most common surgical procedures performed in Western countries. In Sweden, with a population of 10 million inhabitants, approximately 110,000 cataract surgeries are performed annually according to the Swedish Cataract Register (2). Cataract is a major burden for health-care providers around the world. From a public health perspective, it is important to know the prevalence of undiagnosed lens opacities. Identifying modifiable predictors for the development of cataract will make it possible to initiate strategies to delay the need for cataract surgery. From a societal perspective, it is also important to reduce the costs for health care, especially with an ageing population in mind.

Prevalence studies on cataract and its risk factors have been widely reported from various parts of the world in the past decades. The results of three of these studies on people in the age span of 65-74years are presented in Table 1. Clearly, in population studies on lens opacities, prevalence rates are a function of the examination methods and diagnostic criteria used. Several classification and grading systems for lens opacities have been developed, including the Wisconsin Cataract Grading System (6) and the Lens
Opacities Classification System III (LOCS III) (7). In both systems, photographs were taken of the lenses and were compared to a set of standard colour transparencies of lens opacities. In other grading systems, the findings at biomicroscopy were categorized according to a written description of lens changes (3).

Previous studies have identified several risk factors associated with cataract, including smoking $(8,9)$, diabetes (10), sunlight exposure (11), high body mass index (12), steroid use (13), increasing age (14,15), female gender $(16,17)$, myopia $(17,18)$, and pseudoexfoliation in the anterior eye segment $(19,20)$. In a study on the Tierp population, pseudoexfoliation was found to be a predictor for cataract surgery (21).

The aim of this investigation was to report the prevalence of age-related cataract and its risk factors in a population survey in Sweden.

\section{Methods}

\section{The Tierp glaucoma survey}

The Tierp Glaucoma Survey was a population-based study on residents in the municipality of Tierp, south-central Sweden, conducted in 1984-86. Its target population comprised 2,429 people, aged $65-74$ years. The size of the sample was limited 
Table 1. Prevalence of cataract in subjects 65-74years of age in the Framingham Eye Study, the Beaver Dam Eye Study, and the Blue Mountains Eye Study.

\begin{tabular}{llll}
\hline Study & Ref. & \multicolumn{1}{c}{ Method } & Prevalence (\%) \\
\hline Framingham & $(3)$ & Slit-lamp, ophthalmoscopy & Total $18.0^{\mathrm{a}}$ \\
& & & Females 19.3 \\
& & & Males 16.0 \\
Beaver Dam & $(4)$ & Photography & Total $74.3^{\mathrm{b}}$ \\
& & & Females 76.3 \\
Blue Mountains & $(5)$ & Photography & Males 71.4 \\
& & & Total $68.4^{\mathrm{b}}$ \\
& & & Females 73.2 \\
& & & Males 62.2 \\
\hline
\end{tabular}

${ }^{a}$ Only individuals with visual acuity of $20 / 30$ or worse in either eye were examined; including subjects with a history of cataract surgery.

${ }^{b}$ Right eye only.

Table 2. Participation in the population survey in Tierp, by age and gender.

\begin{tabular}{|c|c|c|c|c|c|c|c|c|}
\hline \multirow[b]{3}{*}{ Age } & \multicolumn{4}{|c|}{ Females $(n=429)$} & \multicolumn{4}{|c|}{ Males $(n=409)$} \\
\hline & \multicolumn{4}{|c|}{ Examined } & \multicolumn{4}{|c|}{ Examined } \\
\hline & Yes & (\%) & No & (\%) & Yes & (\%) & No & (\%) \\
\hline $65-69$ years & 209 & (93.3) & 15 & (6.7) & 195 & $(90.7)$ & 20 & (9.3) \\
\hline $70-74$ years & 187 & (91.2) & 18 & (8.8) & 169 & (87.1) & 25 & (12.9) \\
\hline $65-74$ years & 396 & (92.3) & 33 & (7.7) & 364 & (89.0) & 45 & (11.0) \\
\hline
\end{tabular}

to about one-third of the target population. Of the eligible number of 838 residents, 760 (91\%) underwent a detailed eye examination, as described elsewhere (22). The characteristics of participants and non-participants are presented in Table 2.

The study was primarily designed to address the distribution and determinants of open-angle glaucoma. However, a great amount of information was collected, including data on cataract. Briefly, an interview was first held, covering medical and family history. Information was also obtained from medical records. After perimetry, the pupils were dilated to a diameter of at least $3 \mathrm{~mm}$ and biomicroscopy undertaken. The presence of cataract was ascertained based on retroillumination using indirect ophthalmoscopy with lens opacities evident on slit-lamp examination. A grading of the amount of opacities in six stages was also performed. Individuals with definite lens opacities in either eye or those who had undergone cataract surgery in one or both eyes were classified as having cataract. The first stage of lens opacities, described as 'early senile changes' in the Framingham Eye Study (3), was not accepted as definite cataract in the present study.

The study was approved by the Human Subjects Committee at the Faculty of Medicine, Uppsala University and adhered to the tenets of the Declaration of Helsinki. Informed consent was obtained from all participants.

\section{Statistical methods}

Prevalence estimates were adjusted for non-response by applying the frequency of cataract among those who presented for the examination to those who did not, with age and gender strata. Confidence intervals (Cls) for prevalence rates were calculated using the normal approximation to the binomial distribution. Risk factors for cataract expressed as
Table 3. Prevalence of cataract in the population survey in Tierp, by age and gender. ${ }^{a}$

\begin{tabular}{|c|c|c|c|c|}
\hline \multirow[b]{2}{*}{ Age } & \multicolumn{2}{|c|}{ Females $(n=396)$} & \multicolumn{2}{|c|}{ Males $(n=364)$} \\
\hline & Prevalence & $95 \% \mathrm{Cl}$ & Prevalence & $95 \% \mathrm{Cl}$ \\
\hline $65-69$ years & $26.3 \%$ & $20.3-32.2$ & $22.1 \%$ & $16.2-27.9$ \\
\hline $70-74$ years & $45.9 \%$ & $37.8-52.0$ & $30.8 \%$ & $23.8-37.7$ \\
\hline $65-74$ years $^{b}$ & $35.2 \%$ & $28.7-41.8$ & $26.2 \%$ & $19.8-32.6$ \\
\hline
\end{tabular}

odds ratios (ORs) were estimated using $2 \times 2$ tables. Control for age and gender was performed according to Mantel-Haenszel. To simultaneously assess several predictors affecting the risk for cataract, multiple logistic regression analyses were also employed with cataract as the dependent variable.

\section{Results}

\section{Prevalence of cataract}

A total of 234 individuals were found to have cataract, 12 of whom had undergone cataract surgery. The prevalence adjusted for non-response was $31.5 \%$ (95\% Cl 29.4-33.6), $35.2 \%(95 \% \mathrm{Cl} 28.7-41.8)$ in women and $26.2 \%(95 \% \mathrm{Cl}$ 19.8-32.6) in men. Another 116 people had early lens changes in either eye, not classified as cataract. The distribution of cataract by age and gender is shown in Table 3. Cataract was strongly related to increasing age in both women and men.

\section{Prevalence of past cataract surgery}

Twelve subjects had a history of cataract surgery, equal to an unadjusted prevalence of $1.6 \%(95 \% \mathrm{Cl} 0.7-2.5)$. Aphakia in one or both eyes was present in 11 subjects and pseudophakia in either eye in two subjects. In addition, traumatic aphakia was observed in two subjects. Of these, one had age-related cataract in the unaffected eye and was included among the cataract cases.

\section{Risk factors for cataract}

To assess risk factors for cataract, ORs were calculated, adjusted for age and gender. Table 4 provides ORs for potential risk factors. High age, female gender, and myopia increased the risk for cataract, while pseudoexfoliation, current smoking, diabetes mellitus, systemic hypertension, and ischaemic heart disease did not.

The variables mentioned in the previous passage were tested in logistic regression models. The ultimate model included cataract, age as a continuous variable, gender, and myopia. For gender and myopia, the result was almost identical to that of the stratified analyses. Every year of advancing age increased the risk for having cataract with 16\% (OR 1.16; $95 \% \mathrm{Cl} 1.10-1.23)$. Females ran a $52 \%$ higher risk than males. There was no indication of interactions in the models. 
Table 4. Odds ratios for cataract in the population survey in Tierp, adjusted for age and gender.

\begin{tabular}{|c|c|c|c|c|}
\hline Characteristics & & $\begin{array}{l}\text { No. of cases } \\
(n=234)\end{array}$ & $\mathrm{OR}_{\mathrm{M}-\mathrm{H}}$ & $95 \% \mathrm{Cl}$ \\
\hline \multirow[t]{2}{*}{ Age $\geq 70$ years $^{a}$} & No & 98 & 1.00 & \\
\hline & Yes & 136 & 1.93 & $1.41-2.64$ \\
\hline \multirow[t]{2}{*}{ Female gender ${ }^{b}$} & No & 95 & 1.00 & \\
\hline & Yes & 139 & 1.54 & $1.12-2.11$ \\
\hline \multirow[t]{2}{*}{ Pseudoexfoliation, either eye } & No & 189 & 1.00 & \\
\hline & Yes & 45 & 1.12 & $0.75-1.68$ \\
\hline \multirow[t]{2}{*}{ Myopia, either eye } & No & 209 & 1.00 & \\
\hline & Yes & 25 & 2.03 & $1.16-3.56$ \\
\hline \multirow[t]{2}{*}{ Current smoker } & No & 206 & 1.00 & \\
\hline & Yes & 28 & 0.73 & $0.46-1.17$ \\
\hline \multirow[t]{2}{*}{ Diabetes } & No & 201 & 1.00 & \\
\hline & Yes & 33 & 1.25 & $0.79-1.99$ \\
\hline \multirow[t]{2}{*}{ Hypertension, treated } & No & 159 & 1.00 & \\
\hline & Yes & 75 & 1.26 & $0.90-1.78$ \\
\hline \multirow[t]{2}{*}{ Ischaemic heart disease } & No & 195 & 1.00 & \\
\hline & Yes & 39 & 1.18 & $0.77-1.80$ \\
\hline
\end{tabular}

${ }^{a}$ Adjusted for gender.

${ }^{\mathrm{b}}$ Adjusted for age.

$\mathrm{Cl}$ : confidence interval; $\mathrm{OR}_{\mathrm{M}-\mathrm{H}}$ : Mantel-Haenszel adjusted odds ratio.

\section{Discussion}

To the best of our knowledge, only one population-based study has previously been reported on cataract prevalence in Sweden, namely the Skövde Cataract Study (23), with the Tierp study being the second. The study in Skövde used the LOCS III grading system, including people 70-84 years of age. A study like the one in Skövde was also conducted in Oulu County, Finland on people aged $\geq 70$ years (24). Furthermore, a case-control study, with controls chosen from the population register, on the association of cataract with diabetes type 2, was undertaken in Laxå, Sweden (25). In this study, a previous version of the LOCS III system was used.

The prevalence of cataract in Tierp was low compared with the population surveys of Beaver Dam, Wisconsin, US and Blue Mountains, Australia (Table 1). However, in subjects 70-74years of age, the prevalence observed in Tierp was rather close to the prevalence in Oulu (38.2 and 44.6\%, respectively). The age-specific prevalence in the Framingham Eye Study was lower than that in Tierp (18.0 and 31.5\%, respectively). A likely explanation for the difference can be the more conservative inclusion criteria in the Framingham study; only subjects with a visual acuity of $20 / 30$ or worse were classified as having cataract. In the Laxå study, the prevalence of cataract in the control group of people $65-74$ years of age was $72.7 \%$ in females and $78.3 \%$ in males. Although the estimates were based on only 24 and 36 cases, respectively, the prevalence in Laxå seems to have been much higher than that in Tierp.

The reported prevalence rates of cataract vary for several reasons. Firstly, there is no clear-cut definition of when a lens is opaque enough to be classified as cataract. In the present study, a subject was thought to have cataract if lens opacities were observed on retroillumination using indirect ophthalmoscopy, and the findings were evident on slit-lamp biomicroscopy. It is likely that this method underestimates the frequency of cataract compared with photographic methods. A standardized lens opacity grading system, like
LOCS III, should be preferred in population surveys on cataract.

Secondly, the age of the examined population differs between studies. Age is an important risk factor for the development of cataract, as shown in this study. Thirdly, inclusion of subjects with a history of cataract surgery may have an impact on prevalence rates. In the present report, the rates presented for the studies in Framingham, Tierp, Oulu, and Laxå included patients who had undergone surgery, while the rates for Beaver Dam and Blue Mountains did not. Fourthly, an unequal distribution of risk factors for cataract such as exposure to sunlight, smoking habits, and diabetes may affect prevalence rates. Finally, the population survey in Tierp was conducted in 1985-86, while some of the studies referred to in this report were performed more recently. It is not impossible that lifestyle factors have changed over time and thereby modified the prevalence rates.

Consistent with previous research, age was found to be a major risk factor for the development of cataract in both females and males. Accumulated oxidative stress to the lens proteins over the years has been suggested as the primary explanation (26). Furthermore, we confirm findings from previous studies that females are at a higher risk of cataract than males $(16,17)$. It has been suggested that oestrogen plays a role in protecting the lens from oxidative stress (27-29). In this context, decreasing oestrogen levels after menopause would increase the risk for cataract. On the contrary, other studies have concluded that postmenopausal hormone replacement therapy increases the risk for undergoing cataract surgery (30).

The association of age-related cataract with myopia is well-known (17). In most cases, the development of myopia is thought to be a consequence of a shift in refraction in subjects with nuclear lens opacities. However, follow-up studies have demonstrated the possibility of a causal relationship between myopia and cataract, in particular posterior subcapsular cataract (18). With an increasing number of myopic individuals in many countries (31), it is possible that cataract may affect more people and appear earlier in life in the coming years. In the present study, myopia increased the risk for having cataract 2-fold. Unfortunately, adjustment for nuclear opacities could not be performed, which is likely to have biased the result.

Cigarette smoking $(8,9)$ and diabetes (10) are established risk factors for lens opacities. Nevertheless, we were unable to confirm the effect of smoking and diabetes. The sparse number of exposed cases is a likely explanation. Furthermore, we could not verify a relationship between cataract and pseudoexfoliation, reported in other population studies $(19,20)$. Other known risk factors like sunlight exposure (11), high body mass index (12), and steroid use (13) were not examined.

Compared with the population studies in Framingham, Beaver Dam, and Blue Mountains, the Tierp Glaucoma Survey was a small study, limiting its statistical power. However, the high participation rate was a strength of the study. The lack of a standardized grading system of cataract, leading to 
potential misclassification of disease, was a limitation of the study. Another weakness was that the type of cataract was not registered in the population survey. There are evidence that cortical and nuclear lens opacities do not share the same modifiable risk factors $(17,32)$. To estimate the lifetime risk of cataract surgery in this cohort of older adults, a follow-up study is in progress.

\section{Conclusion}

Nearly one-third of the participants in the Tierp Glaucoma Survey were found to have lens opacities, or had undergone cataract surgery, making cataract a frequent disorder of older age. Nevertheless, the age-specific prevalence was low compared with studies using standardized grading systems based on photography. The study provided further evidence that increasing age, female gender, and myopia are associated with cataract.

\section{Disclosure statement}

The authors report no conflicts of interest.

\section{Funding}

The Swedish Medical Research Council, Crown Princess Margaretha's Foundation for the Visually Impaired, the Glaucoma Research Fund at the Department of Ophthalmology, Uppsala University Hospital, and Uppsala County Council provided financial support for this study.

\section{Notes on contributors}

Magnus Hugosson is a MD at the Department of Ophthalmology, University Hospital, Uppsala, Sweden.

Curt Ekström, MD, PhD, is a senior researcher at the Department of Neuroscience, Ophthalmology, Uppsala University, Uppsala, Sweden.

\section{ORCID}

Curt Ekström (iD http://orcid.org/0000-0002-8265-6518

\section{References}

1. Khairallah $M$, Kahloun $R$, Bourne R, Limburg $H$, Flaxman SR, Jonas $\mathrm{JB}$, et al. Number of people blind or visually impaired by cataract worldwide and in world regions, 1990 to 2010. Invest Ophthalmol Vis Sci. 2015;56:6762-9.

2. National Quality Register for Cataracts. 2019 Aug 8. Available at: http://kvalitetsregister.se/englishpages/findaregistry/registerarkivenglish/nationalqualityregistryforcataracts.2150.html

3. Leibowitz HM, Krueger DE, Maunder LR, Milton RC, Kini MM, Kahn $\mathrm{HA}$, et al. The Framingham eye study monograph. Surv Ophthalmol. 1980;24:350-65.

4. Klein BEK, Klein R, Linton KLP. Prevalence of age-related lens opacities in a population: The Beaver Dam Eye Study. Ophthalmology 1992;99:546-52.

5. Mitchell P, Cumming RG, Attebo K, Panchapakesan J. Prevalence of cataract in Australia: the Blue Mountains eye study. Ophthalmology 1997;104:581-8.

6. Klein BE, Klein R, Linton KL, Magli YL, Neider MW. Assessment of cataracts from photographs in the Beaver Dam Eye Study. Ophthalmology 1990;97:1428-33.
7. Chylack LT, Jr, Wolfe JK, Singer DM, Leske MC, Bullimore MA Bailey IL, et al. The lens opacities classification system III. The Longitudinal Study of Cataract Study Group. Arch Ophthalmol. 1993;111:831-6.

8. Hiller R, Sperduto RD, Podgor MJ, Wilson PW, Ferris FL 3rd, Colton $\mathrm{T}$, et al. Cigarette smoking and the risk of developing lens opacities. The Framingham studies. Arch Ophthalmol. 1997; 115:1113-8.

9. Lindblad BE, Håkansson N, Svensson H, Philipson B, Wolk A. Intensity of smoking and smoking cessation in relation to risk of cataract extraction: a prospective study of women. Am J Epidemiol. 2005;162:73-9.

10. Klein BE, Klein R, Lee KE. Diabetes, cardiovascular disease, selected cardiovascular disease risk factors, and the 5-year incidence of age-related cataract and progression of lens opacities: The Beaver Dam Eye Study. Am J Ophthalmol. 1998;126:782-90.

11. West SK, Duncan DD, Muñoz B, Rubin GS, Fried LP, BandeenRoche $\mathrm{K}$, et al. Sunlight exposure and risk of lens opacities in a population-based study: the Salisbury Eye Evaluation project. JAMA 1998;280:714-8.

12. Hiller R, Podgor MJ, Sperduto RD, Nowroozi L, Wilson PW, D'Agostino RB, et al. A longitudinal study of body mass index and lens opacities. The Framingham Studies. Ophthalmology 1998;105: 1244-50.

13. Wang JJ, Rochtchina E, Tan AG, Cumming RG, Leeder SR, Mitchell $P$. Use of inhaled and oral corticosteroids and the long-term risk of cataract. Ophthalmology 2009;116:652-7.

14. Sasaki $H$, Jonasson $F$, Kojima $M$, Katoh N, Ono M, Takahashi N, et al. The Reykjavik Eye Study-prevalence of lens opacification with reference to identical Japanese studies. Ophthalmologica. 2000;214:412-20.

15. Xu L, Cui T, Zhang S, Sun B, Zheng $Y, H u A$, et al. Prevalence and risk factors of lens opacities in urban and rural Chinese in Beijing. Ophthalmology 2006;113:747-55.

16. Hiller R, Sperduto RD, Ederer F. Epidemiologic associations with nuclear, cortical, and posterior subcapsular cataracts. Am J Epidemiol. 1986;124:916-25.

17. McCarty CA, Mukesh BN, Fu CL, Taylor HR. The epidemiology of cataract in Australia. Am J Ophthalmol. 1999;128:446-65.

18. Younan C, Mitchell P, Cumming RG, Rochtchina E, Wang JJ. Myopia and incident cataract and cataract surgery: the Blue Mountains Eye Study. Invest Ophthalmol Vis Sci. 2002;43: 3625-32.

19. Hiller R, Sperduto RD, Krueger DE. Pseudoexfoliation, intraocular pressure, and senile lens changes in a population-based survey. Arch Ophthalmol. 1982;100:1080-2.

20. Arvind H, Raju P, Paul PG, Baskaran M, Ve Ramesh S, George RJ, et al. Pseudoexfoliation in South India. Br J Ophthalmol. 2003;87: 1321-3.

21. Ekström C, Botling Taube A. Pseudoexfoliation and cataract surgery: a population-based 30-year follow-up study. Acta Ophthalmol. 2015;93:774-7.

22. Ekström C. Prevalence of open-angle glaucoma in central Sweden. The Tierp Glaucoma Survey. Acta Ophthalmol Scand. 1996;74: 107-12.

23. Östberg A, Löth A, Gustafson D, Lindblom B. Skövde Cataract study: I. Prevalence of lens opacities in a Swedish community. Ophthalmology 2006;113:970-5.

24. Hirvelä $H$, Luukinen $H$, Laatikainen L. Prevalence of risk factors of lens opacities in the elderly in Finland. Ophthalmology 1995;102: 108-5.

25. Olafsdottir E, Andersson DKG, Stefánsson E. The prevalence of cataract in a population with and without type 2 diabetes mellitus. Acta Ophthalmol. 2012;90:334-40.

26. Jaskiran K, Kukreja S, Kaur A, Malhotra N, Kaur R. The oxidative stress in cataract patient. J Clin Diagn Res 2012;6:1629-32.

27. Klein BE, Klein R, Ritter LL. Is there evidence of an estrogen effect on age-related lens opacities? The Beaver Dam Eye Study. Arch Ophthalmol. 1994;112:85-91. 
28. Worzala $K$, Hiller $\mathrm{R}$, Sperduto RD, Mutalik $\mathrm{K}$, Murabito JM, Moskowitz $M$, et al. Postmenopausal estrogen use, type of menopause, and lens opacities: the Framingham studies. Arch Intern Med. 2001;161:1448-54.

29. Škiljić D, Petersen A, Jan-Olof Karlsson J-O, Behndig A, Nilsson S, Zetterberg $M$. Effects of $17 \beta$-estradiol on activity, gene and protein expression of superoxide dismutases in primary cultured human lens epithelial cells. Curr Eye Res. 2018;43:639-46.

30. Ejdervik Lindblad $B E$, Håkansson $\mathrm{N}$, Philipson $\mathrm{B}$, Wolk A. Hormone replacement therapy in relation to risk of cataract extraction: a prospective study of women. Ophthalmology 2010; 117:424-30.

31. Holden BA, Fricke TR, Wilson DA, Jong M, Naidoo KS, Sankaridurg $P$, et al. Global prevalence of myopia and high myopia and temporal trends from 2000 through 2050. Ophthalmology 2016;123: 1036-42.

32. Arnarsson A, Jonasson F, Sasaki H, Ono M, Jonsson V, Kojima M, et al. Reykjavik Eye Study Group. Risk factors for nuclear lens opacification: the Reykjavik Eye Study. Dev Ophthalmol. 2002;35: 12-30. 